\title{
ANGPTL4 Negatively Regulate the Progression of Osteosarcoma by Remodeling Branched-Chain Amino Acid Metabolism
}

\section{Shanyi Lin}

Department of Orthopaedic Surgery, Shanghai Jiao Tong University Affiliated Sixth People's Hospital

\section{Yu Miao}

Department of Orthopaedic Surgery, Shanghai Jiao Tong University Affiliated Sixth People's Hospital

\section{Xu Zheng}

Department of Orthopaedic Surgery, Shanghai Jiao Tong University Affiliated Sixth People's Hospital

\section{Yang Dong}

Department of Orthopaedic Surgery, Shanghai Jiao Tong University Affiliated Sixth People's Hospital

\section{Qingcheng Yang}

Department of Orthopaedic Surgery, Shanghai Jiao Tong University Affiliated Sixth People's Hospital

\section{Quanjun Yang}

Department of Pharmacy, Shanghai Jiao Tong University Affiliated Sixth People's Hospital

\section{Jun Xu}

Department of Orthopaedic Surgery, Shanghai Jiao Tong University Affiliated Sixth People's Hospital

\section{Shumin Zhou}

Institute of Microsurgery on Extremities, Shanghai Jiao Tong University Affiliated Sixth People's Hospital

Ting Yuan ( $\nabla$ terrenceyuan@gmail.com )

Department of Orthopaedic Surgery, Shanghai Jiao Tong University Affiliated Sixth People's Hospital

\section{Research Article}

Keywords: ANGPTL4, Osteosarcoma, BCAAs, mTOR

Posted Date: December 30th, 2021

DOI: https://doi.org/10.21203/rs.3.rs-1209880/v1

License: (c) (1) This work is licensed under a Creative Commons Attribution 4.0 International License. Read Full License

Version of Record: A version of this preprint was published at Cell Death Discovery on April 23rd, 2022. See the published version at https://doi.org/10.1038/s41420-022-01029-x. 


\section{Abstract}

\section{Background}

Angiopoietin-like-4 (ANGPTL4), a secreted glycoprotein that is mainly recognized as a regulator in lipid metabolism, now, is implied in the regulation of the growth and metastasis of various carcinomas. However, less is known about its functions in the progression of sarcomas, let alone osteosarcoma (OS), which is the most common malignant diagnosed in musculoskeletal system.

\section{Methods}

The expression of ANGPTL4 in clinical OS samples and cell lines paired with their controls were analyzed in both mRNA and protein levels. Cell functional analysis including proliferation and colony formation were carried out to detect the roles ANGPTL4 takes in the progress of OS using stable ANGPTL4 overexpression and knockdown HOS/MNNG cell lines. The RNA-Seq and bioinformatics analysis were then employed to discover the BCAA metabolism related signaling which is involved in ANGPTL4 functioning on HOS/MNNG cell growth. Furthermore, BCAAs content measurement, and BCATs rescue experiments were performed to confirm the BCAA/mTOR signaling axis that ANGPTL4 triggered in HOS/MNNG cells. Finally, a xenograft mouse model was carried out to further verify the ANGPTL4 /BCAA/mTOR signaling axis discovered.

\section{Results}

We found that the expression of ANGPTL4 is reduced in clinical OS tissues and cell lines compared to cancellous bone tissues and BMSCs, respectively. The knockdown of ANGPTL4 in HOS/MNNG cells results in enhanced cell growth and clone formation. Moreover, BCAA/mTOR signaling axis were discovered to be triggered by ANGPTL4 down regulation in HOS/MNNG cell using RNA-seq. It was also verified that the accumulation of BCAAs activates the mTOR signaling pathway, and in turn promotes HOS/MNNG cell growth using BCAAs content measurement, and BCAT inhibition. Finally, the IHC results of xenograft mouse model also confirmed this ANGPTL4/BCAA/mTOR signaling axis in vivo.

\section{Conclusions}

Taken together, our results demonstrate that the expression of ANGPTL4 were negatively related to OS progress. Moreover, it was found the down-regulation of ANGPTL4 promoted OS cell growth via BCAAs/mTOR axis.

\section{Introduction}

Osteosarcoma (OS) is a major health burden and cause of cancer-related death in adolescents worldwide (1). Patients diagnosed with OS face the threat of amputation and even death after tumor cells metastasize to the lungs (2). Currently, the treatment of OS includes complete tumor removal and two rounds of chemotherapy (i.e., preoperative and postoperative 
chemotherapy), which are continuous for at least 6 months (2). However, such systematic therapies could only cure $60 \% \sim 70 \%$ of patients. The 5 -year survival rate of OS patients has not improved, although surgical techniques have improved considerably in recent decades $(2,3)$. To improve the prognosis of OS patients, clinical trials of some second-line or third-line drugs are in full flow $(4,5)$. In order to develop more drugs against OS, it is important to fully understand the mechanisms underlying the occurrence and progression of OS.

Angiopoietin-like proteins (ANGPTLs) are a protein family that includes eight members characterized as structurally similar to angiogenin (6). ANGPTL4, a member of the ANGPTL family that is mainly found in the liver, adipose tissue, and skeletal muscle, is well known as an inhibitor of lipoprotein lipase $(\mathrm{LPL})(7,8)$. Due to the organ expression bias of ANGPTL4, it was initially regarded as merely a metabolic regulator that maintains metabolic homeostasis $(8,9)$. Recently, ANGPTL4 has also been detected in diverse tumor cells and associated with malignant phenotypes. Chen et al. found that the expression of ANGPTL4 was higher in gastric cancer cells and that the knockdown of ANGPTL4 could suppress the development of gastric cancer (10). However, Cai et al. demonstrated that ANGPTL4 was a favorable prognostic factor in breast cancer and that upregulation of ANGPTL4 induced the suppression of adhesion and migration in cancer cells (11). Besides, ANGPTL4 is also involved in the temozolomide resistance of glioblastoma by promoting cancer stemness (12). Although researchers have started to explore the role of ANGPTL4 in OS (13), the underlying fine molecular mechanism remains barely unclear.

In the present study, a clearly lower expression of ANGPTL4 was found in both clinical OS tissue samples and OS cell lines, compared to that found in normal control groups. Knockdown of ANGPTL4 leads to the acceleration of proliferation in OS cells. RNA-Seq analysis suggests that ANGPTL4 expression results in the remodeling of branched-chain amino acid (BCAA) metabolism. BCAAs are essential amino acids (leucine, isoleucine and valine) which play a crucial role in protein synthesis and energy, and are indispensable for cell growth $(14,15)$. When the accumulation of BCAAs was up-regulated in OS cell lines due to knockdown of ANGPTL4, the mTOR signaling pathway was activated, which results in enhanced growth of OS cells. Taken together, our results indicate the low-expression of ANGPLT4 promotes the progression of OS via mTOR signaling by remodeling BCAA metabolism.

\section{Materials And Methods \\ 2.1 Clinical samples}

The clinical specimens were collected from the Department of Bone Oncology, and Department of Emergency, Shanghai Jiao Tong University Affiliated Sixth People's Hospital, including osteosarcoma tissue and normal cancellous bone tissue. The information of patients both in osteosarcoma group and control group were shown in Supplementary table 1 ana Supplementary table 2. Ethical approval was obtained from the Ethics Committee of Shanghai Sixth People's Hospital (YS-2016-064, February 24, 2016). 


\subsection{RNA extraction and real-time quantitative PCR (RT- qPCR)}

Total RNA from both clinical specimens and cell lines was extracted by TRIzol reagent (Invitrogen, USA) and then reverse transcribed to CDNA according to the instructions of a cDNA Synthesis Kit (Invitrogen, USA). The relative expression levels of genes were measured on an ABI Prism 7900HT real-time system (Applied Biosystems) and calculated by the $2^{-\Delta \Delta C t}$ approach. All primers were shown in Supplementary Table 3.

\subsection{Western blot and reagent}

Total proteins were extracted by RIPA solution (EpiZyme, PC102) according to standard procedures. Then, the collected proteins were separated by electrophoresis and transferred to a PVDF membrane. The nonspecific binding sites of the PVDF membrane were blocked with $5 \%$ milk at room temperature (RT) for $60 \mathrm{~min}$. Target proteins were detected by incubating in the primary antibody solution at $4{ }^{\circ} \mathrm{C}$ overnight. Finally, protein bands were detected by chemiluminescence. All Primary antibodies were shown in Supplementary Table 4.

\subsection{Cell culture}

Human MNNG/HOS (MNNG is used instead in the following), U2OS, and MG63 were purchased from American Type Culture Collection (ATCC). Human BMSCs were harvested from patients with open fractures who underwent debridement at Shanghai Jiao Tong University Affiliated Sixth People's Hospital. The culture media for MNNG, MG63, and U2OS, and BMSCs were DMEM (Corning, USA), RPMI 1640 (Corning, USA), and a-MEM (Corning, USA), respectively. All culture media were supplemented with $10 \%$ fetal bovine serum (Gibco, USA). All cells were cultured at a $37^{\circ} \mathrm{C}$ atmosphere containing $5 \% \mathrm{CO} 2$.

\subsection{Stable cell line construction}

For stable cell line construction, HEK293T cells were co-transfected with lentivirus packing vectors and LV shuttle plasmids containing full-length ANGPTL4 and siRNA against ANGPTL4. Forty-eight hours later, the supernatant containing lentivirus was collected by centrifugation at $400 \mathrm{~g}$ for $10 \mathrm{~min}$, purified and titer determined. Then, the lentivirus was added to the culture medium of MNNG cells at an MOI of 10.0 to infect the cells. After 72 hours, the culture medium was changed to a new medium that contained puromycin at a concentration of $1.0 \mu \mathrm{g} / \mathrm{ml}$ to select the positive cells. Finally, stable cells overexpressing (MNNG-A4) and knockdown ANGPTL4 (MNNG-siA4) were verified by RTqPCR and WB. All the information of the vector and sequences of full-length ANGPTL4 and siRNA against ANGPTL4 has been provided in the Supplementary Table 5 . 


\subsection{Cell proliferation assay}

Real-time cellular analysis (RTCA) (ACEA Biosciences, USA) was used to evaluate the cell proliferation ability (16). First, the baseline value was measured in $100 \mu \mathrm{l}$ of culture medium which pre-incubated at 37 ${ }^{\circ} \mathrm{C}$ in a cell incubator for 1 hour. Then, the cells were seeded into wells at a density of $2.0 \times 10^{3}$ cells per well. The attachment and proliferation of cells were measured by the RTCA system for 6 hours and 168 hours, respectively.

\subsection{Colony formation assay}

The cells were seeded into a 6-well plate to ensure that there were 1000 cells per well. After incubation for 14 days, the cells were fixed with $4 \%$ paraformaldehyde (PFA), and then the cells were immersed in crystal violet for half an hour. The cells in each well were photographed and recorded, and colonies containing more than 50 cells were counted by Image J software.

\subsection{Subcutaneous tumor model}

Female nude mice ranging from 4-6 weeks were purchased from the Laboratory Animal Research Center of Shanghai Sixth People's Hospital, and all operations were approved by the Animal Research Committee of Shanghai Sixth People's Hospital. After anesthesia by pentobarbital sodium, $200 \mu \mathrm{l}$ of cell suspension containing $1 \times 10^{6}$ cells were injected into the nude mouse flank (17). Tumors were measured by researchers until the longest diameter of the largest tumor reached $200 \mathrm{~mm}$. The volume of tumors was calculated as length $(\mathrm{mm}) \times$ width $(\mathrm{mm})^{2} / 2$.

\subsection{RNA-seq and analysis}

TRIZOL (Invitrogen, USA) was used to extract total RNA from MNNG-A4, MNNG-siA4, and control cell lines. Then, standard guidelines were followed to construct paired-end libraries with a TruSeq ${ }^{T M}$ RNA Sample Preparation Kit (Illumina, USA). The mRNA is cleaved into small pieces and reverse transcribed into first strand cDNA. Then, DNA polymerase I and RNase $\mathrm{H}$ were used to generate second strand $c D N A$. These cDNAs then undergo the addition of a single ' $A$ ' base and then ligation of the adapters. The products were purified and enriched with PCR to create the final cDNA library. Library construction and sequencing were performed by Sinotech Genomics Co., Ltd. (Shanghai, PRC). Differentially expressed genes (DEGs) were selected based on a false discovery rate (FDR) less than $5 \%$ and changed expression higher than 1.5 -or lower than 0.67 -fold. All cell lines were tested three times.

\subsection{Branched Chain Amino Acids (BCAAs) Assay}


Cells $\left(2 \times 10^{6}\right)$ were harvested from a T75 cell culture flask. Cells were lysed, and the levels of BCAAs were measured by a BCAA assay kit according to the manufacturer's instructions (SigmaAldrich, USA). Briefly, cells were lysed in $100 \mathrm{~mL}$ of cold BCAA assay buffer to obtain the lysate, 10 $\mu \mathrm{l}$ of lysate was added to a 96-well plate, and BCAA buffer was added to bring the volume to $50 \mathrm{~mL}$. Next, $50 \mu \mathrm{l}$ reaction mixes containing $46 \mu \mathrm{l}$ assay buffer, $2 \mu \mathrm{l}$ BCAA enzyme mix, and $2 \mu \mathrm{l}$ WST substrate mix were added to each well. The blank wells contained the cell lysate, BCAA assay buffer, and substrate mix but omitted the enzyme. Then, the reaction was incubated for 30 minutes at room temperature, and the absorbance was measured at $450 \mathrm{~nm}$ (A450). The blank absorbances were subtracted from lysate absorbances. A standard curve was generated through the above method, but the cell lysate was changed with a leucine standard. Each sample was measured in duplicate.

\section{$2.11 \mathrm{Immunohistochemical} \mathrm{(IHC)} \mathrm{analysis}$}

The clinical OS specimens and OS tissues excised from the subcutaneous tumor model were embedded in paraffin and then cut into $4 \mu \mathrm{m}$ sections and deparaffinized. The sections were blocked with $5 \%$ bovine serum albumin (BSA) at $37^{\circ} \mathrm{C}$ for $30 \mathrm{~min}$ after antigen retrieval. After that, specific primary antibodies were added to the samples and incubated overnight at $4{ }^{\circ} \mathrm{C}$. Then, the cells were washed three times with PBS and incubated with HRP-linked anti-IgG at $37^{\circ} \mathrm{C}$ for $30 \mathrm{~min}$. Washing by PBS again and stained in DAB for 10 min. Finally, the samples were counterstained, dehydrated, covered with cover glass and photographed with DM6B (Leica, BRD).

\subsection{Statistical analyses}

The data were analyzed by SPSS 25.0 software and presented as the mean \pm SD. The differences between experimental and control groups were analyzed by two-tailed Student's $t$ test while the differences between tumor tissues group and nontumor tissues group of clinical samples were analyzed by Welch's t test. ns means $\mathrm{P}>0.05$, * means $\mathrm{P}<0.05$, ** means $\mathrm{P}<0.01$, and $* \star \star$ means $\mathrm{P}<0.001$.

\section{Results}

\subsection{ANGPTL4 was expressed at low levels in clinical OS samples and OS cells}

To investigate the role of ANGPTL4 in the development of OS, the expression level of ANGPTL4 in clinical OS tissue samples and control samples (normal cancellous bone) was first evaluated in mRNA level. As shown in Fig. 1A, there was a clear lower expression pattern of ANGPTL 4 found in OS tissues than those in adjacent normal bone tissues. Then, we further analyzed the expression of ANGPTL4 protein by immuno-histochemical (IHC) staining in both OS tissue sections and adjacent nontumor sections. Following the same pattern as the RT-qPCR results, OS tissues also showed 
less positive area of ANGPTL4 staining compared to the control sections (Fig. 1D). Besides, the expressions of ANGPTL4 were verified in three OS cell lines: HOS/MNNG (short for MNNG in the following text), U2OS, and MG63 and one control cell: BMSC in both mRNA and protein levels, too. As a result, the mRNA and protein expressions of ANGPTL4 in MNNG, U2OS, and MG63 cells were $0.52 \pm 0.02$, $0.69 \pm 0.04,0.73 \pm 0.02$, and $0.61 \pm 0.02,0.62 \pm 0.04,0.67 \pm 0.06$, folds lower than that in BMSC, respectively (Fig. 1B, C). These data confirmed that OS tissues and cell lines had lower expressions of ANGPTL4 than those in cancellous bones and BMSCs in both mRNA and protein levels.

\subsection{ANGPTL4 down-regulation promotes the growth of OS cells in vitro}

Our previous results suggests that ANGPTL4 may have a negative correlation with the progress of OS. Therefore, the question how ANGPTL4 negatively affecting OS growth is raised. Among the three OS cell lines we tested, MNNG cells showed the lowest ANGPTL4 expression compared to BMSC cells. Herein, the MNNG cell was selected as an in vitro model for validation. Using lentivirus system, we constructed ANGPTL4 up-regulation (MNNG-A4) and down-regulation (MNNG-siA4) stable OS cell lines, as well as their control cell lines (A4Control and siControl) in order to further detect the biological role ANGPTL4 takes in MNNG growth. As shown in Fig. 2A-D, the successful construction of MNNG-A4 (ANGPTL4 upregulation stable OS cell lines) and MNNG-siA4 (ANGPTL4 down-regulated stable OS cell lines) were confirmed in both mRNA and protein levels by RT-qPCR and western blotting.

We then evaluated the cell proliferations of these two ANGPTL4-regulated MNNG cell lines with their control cells by RTCA assay (16). The results showed that the cell growth was significantly suppressed in the MNNG cells overexpressing ANGPTL4 (Ratio of MNNG-A4 vs A4Control was $0.30 \pm 0.01,0.22 \pm 0.01$, $0.21 \pm 0.02$, and $0.20 \pm 0.01$ at $48 \mathrm{~h}, 72 \mathrm{~h}, 96 \mathrm{~h}$, and $120 \mathrm{~h}$ ). Whereas, the knockdown of ANGPTL4 in MNNG cells enhanced their growth (Ratio of MNNG-siA4 vs siControl was $1.23 \pm 0.04,1.43 \pm 0.09,1.75 \pm 0.30$, and $1.40 \pm 0.15$ at $48 \mathrm{~h}, 72 \mathrm{~h}, 96 \mathrm{~h}$, and $120 \mathrm{~h}$ ) (Fig. $2 \mathrm{E}-\mathrm{H}$ ). Accordingly, the colony formation assays were also consistent with the cell proliferation assays (Ratio of MNNG-A4 vs A4Control and MNNG-siA4 vs siControl was $0.77 \pm 0.04$ and $1.39 \pm 0.04$, respectively) (Fig. $2 \mathrm{l}-\mathrm{L}$ ). Taken together, these results demonstrated that the expression of ANGPTL4 showed a negative correlation with the growth of OS cells in vitro.

\subsection{ANGPTL4 actives mTOR signal pathway via remodeling branched-chain amino acid (BCAA) metabolism}

In the interest of exploring the underlying mechanism of how ANGPTL4 affects the cell growth in MNNG cells, we employed mRNA sequencing to discover the changed signaling cascades by identifying differentially expressed genes (DEGs) between ANGPTL4-regulated MNNG cells and their control cells. The DEGs numbers are shown in Fig. 3A and 3B based on an cut off value that changed expression 
higher than 1.5-or lower than 0.67-fold. There were 6,087 DEGs (2,545 up-regulated, 3,542 downregulated) between the control and the ANGPTL4-upregulated cell line, whereas in the ANGPTL4downregulated group the number was 6,957 (3,356 up-regulated, 3,601 down-regulated).

Then the Kyoto Encyclopedia of Genes and Genomes (KEGG) analysis was firstly performed on these DEGs to more detail the underlying mechanisms. As presented in Fig. 3C, 3D and Fig. S1, the results suggested that the DEGs in both ANGPTL4-regulated MNNG (overexpression and knockdown) cells were highly related to the metabolism of Branched-Chain Amino Acid (BCAA). There were 22 out of 46 genes have been found to be different expressed in this gene cluster, which were shown in Fig. 3E. To better understand the molecular action mechanism of these genes, we then introduced these genes into STRING (https://string-db.org/) database to constructing the protein-protein interaction network. As Fig. 3F showed, these genes are closely clustered together and form the complex network, which shows the precise inter-regulatory relationship of these genes are highly involved in BCAAs metabolism.

To verify the results of RNA-seq, we then detected the expressions of these genes in the ANGPTL4regulated MNNG cells. The RT-qPCR results were in accord with the RNA-seq. As shown in Fig. 4A and $4 \mathrm{~B}$, the expression of $H M G C L$ and $A B A T$ were upregulated while $A H U, A O X-1$, ALDH6A1, BCKDHA, BCKDHB, IL 4I-1, SDSL, and ACADS were downregulated in MNNG-A4 cells. Meanwhile, we also observed a opposite trend of these genes in MNNG-siA4 cells. Furthermore, the western blotting results also verified that the protein expression levels of some important genes (AOX-1, BCKDHA, IL4I-1, and HMGCL) were consistent with their mRNA levels (Fig. 4C). To further confirm our hypothesis, we also measured the BCAAs concentrations in both MNNG-A4 and MNNG-siA4 cells and their control cells. As shown in Fig. 4D, MNNG-A4 cells displayed a marked decrease of BCAAs compared with control cells (MNNG-A4 vs A4Control was $0.09 \pm 0.01 \mathrm{nmol} / \mathrm{ul}$ vs $0.12 \pm 0.01 \mathrm{nmol} / \mathrm{ul}$ ), and the expression of BCAAs content was significantly increased in MNNG-siA4 cells (MNNG-siA4 vs siControl was $0.17 \pm 0.01$ vs 0.10 $\pm 0.01)$.

According to the previously studies, BCAAs could both be catabolized into BCKAs and entered the TCA cycle $(14,15)$, and activate the mTOR signaling and promote cell growth (18). Thus, we then examined the activation of mTOR signaling in ANGPTL-regulated MNNG cells and control cells. As shown in Fig. 4E, the phosphorylation levels of mTOR together with the downstream effectors S6 kinase and S6 were upregulated in MNNG-siA4 cells, while a opposite expression patterns found in MNNG-A4 cells compared with control cells. Overall, these data demonstrated that low expression of ANGPTL4 in MNNG cells increased the concentration of BCAAs, which in turn activated the mTOR pathway and promoted the progression of MNNG cells.

\subsection{The inhibition of BCATs attenuated the elevated proliferation in ANGPTL4 down-regulated MNNG cells}


As mentioned above, when BCAAs imported into the cells, they are converted to branched-chain a-keto acids (BCKAs) firstly, by branched-chain amino acid transaminases (BCATs). This catalytic reaction was reversible, which also production of BCAAs via BCKAs (15). Though the RNA-seq results did not point out the expression levels of BCATs were regulated by ANGPTL4 directly, the previous data we got still held a possibility that ANGPTL4 affected BCAA metabolism via enhancing the activities of BCATs. To verify our hypothesis, we treated MNNG-siA4 and siControl cells with a BCATs inhibitor: BCATc inhibitor 2 (19). As expected, it was showed that $5 \mu \mathrm{M}$ of BCATc inhibitor 2 reduced the cell proliferation (Ratio of MNNGsiA4+inhibitor vs siControl+inhibitor was $0.63 \pm 0.24,0.56 \pm 0.13,0.58 \pm 0.08$, and $0.68 \pm 0.07$ at $48 \mathrm{~h}$, $72 \mathrm{~h}, 96 \mathrm{~h}$, and $120 \mathrm{~h}$, respectively) and colony formation (Ratio of MNNG-siA4+inhibitor vs siControl+inhibitor was $0.96 \pm 0.02$ ) of MNNG-siA4 cells (Fig. 5A-D). Mechanismly, the expression levels of BCAA metabolism signaling pathway related proteins (AOX-1, BCKDHA, IL4I-1, and HMGCL) were also reversed in MNNG-siA4 and siControl cells after $5 \mu \mathrm{M}$ of BCATs inhibitor treatment (Fig. 5E, F). Moreover, the BCAA concentrations were also measured in MNNG-siA4 and siControl cells after BCATs inhibitor treatment. The results showed that the high level of BCAA induced by knockdown of ANGPTL4 in OS cells was reduced by BCATc inhibitor 2 (MNNG-siA4+inhibitor vs siControl+inhibitor was $0.08 \pm 0.01 \mathrm{nmol} / \mathrm{ul}$ and $0.13 \pm 0.01 \mathrm{nmol} / \mathrm{ul}$, respectively) (Fig. $5 \mathrm{G}$ ). In summary, these data confirmed that downregulation of ANGPTL4 promotes MNNG growth by regulating the metabolism of BCAAs.

\subsection{ANGPTL4 attenuates OS progress via BCAA/mTOR axis in vivo}

Based on the in vitro results we obtained, the downregulation of ANGPTL4 leads to the accumulation of BCAAs in cells by enhancing the activities of BCATs, which triggers mTOR signaling pathway, and ultimately promotes the proliferation of OS cells. In order to examine this signaling axis in vivo, we then carried out a nude mice subcutaneous implantation tumor model. Briefly, $1 \times 10^{6}$ cells were injected into the nude mouse flank. When the longest diameter of the largest tumor reached $200 \mathrm{~mm}$, the tumors were then excised, measured and recorded. After embedded and cut into sections, the OS cell implants were detected with antibodies against ANGPTL4, BCAT1, BCKDHA, p-mTOR, and p-S6. The intensities of these IHC staining targeting above proteins was analyzed, calculated and perform statistics (Fig. 6A).

As shown in Fig. 6B, the growth of OS implantations was significantly promoted in MNNG-siA4 cells (Ratio of MNNG-siA4 vs siControl was $3.01 \pm 1.40$ ); while the MNNG cells overexpressing ANGPTL4 showed an attenuated growth (Ratio of MNNG-A4 vs A4Control was $0.40 \pm 0.30$ ) (Fig. 6B). Then, we detected the ANGPTL4/BCAA/mTOR signaling in the OS tissues obtained from these nude mouse models by IHC staining (Fig. 6C). As shown in Fig. 6C, ANGPTL4 down-regulated OS tissue presented high expressions of these proteins (Ratio of MNNG-siA4 vs siControl for ANGPTL4, BCAT1, BCKDHA, pmTOR, and p-S6 was $0.87 \pm 0.03,1.07 \pm 0.02,1.14 \pm 0.02,1.57 \pm 0.15$, and $1.07 \pm 0.01$ ). While in the ANGPTL4 up-regulated tissue, the expression patterns of these key proteins showed an opposite pattern except BCAT1 (Ratio of MNNG-A4 vs A4Control for ANGPTL4, BCAT1, BCKDHA, p-mTOR, and p-S6 was $1.28 \pm 0.02,0.92 \pm 0.04,0.83 \pm 0.02,0.92 \pm 0.03$, and $0.90 \pm 0.01$ ) (Fig. 6D). 


\section{Discussion}

BCAAs, the essential amino acids for humans, are important participants in metabolic regulation. The BCAAs were crucial in protein synthesis and energy supply which laid a strong foundation for unrestricted division and durably growth of tumor cells (15) (20). However, it seems not all tumor cells possess the same pattern of BCAA metabolism. The increased level of circulating BCAAs as a result of the protein breakdown or BCKAs aminating could be observed in cancer patients with pancreatic adenocarcinoma and leukemia $(21,22)$. These do not imply that the BCAAs level was always elevated in cancer patients. Indeed, a decrease in circulating BCAAs caused by increased tumor cell uptake and breakdown has also been found in lung tumor cells (23). Mayers et al. attributed such distinct phenotypes to the difference in mutations and the origin of cancer tissues (23). Furthermore, BCAAs were recently regarded as an upstream signal input of the mTOR signaling pathway, which activates the pathway regulating the growth and proliferation of cancer cells (18) (24-26). Several recent studies have discovered that the accumulation of BCAAs in cancer cells due to the reduction of catabolism may enhance the activity of mTOR signaling pathway and promote the progression of cancers $(21,22)(27-29)$. However, less is known on the functions of BCAA in the progression of osteosarcomas.

In our present study, we showed the possible that ANGPLT4 triggers BCAA/mTOR signaling axis in OS cells. Knockdown of ANGPTL4 in OS cell lines changed the metabolism of BCAAs and then enhanced the accumulation of BCAAs in these cells. This led to the activation of the mTOR signaling pathway, which result in the promotion the growth of OS cell (Fig. 7). Our findings provide further evidence for the theory that BCAA metabolism was implicated with the progress of various tumors and confirmed that BCAA metabolism can be subject to regulation by ANGPTL4.

Although the role of ANGPTL4 played in the metabolism regulation was compelling, the impacts of ANGPTL4 in the progression of the tumor were confusing. Previous studies discovered that ANGPTL4 could have a higher expression in tumor patients and evoke malignant phenotype of tumor cells, i.e., proliferation, migration, and drug resistant $(10)(12)(30,31)$. However, some subsequent studies demonstrated that ANGPTL4 showed an anti-angiogenesis as well as antitumor cell invasion and migration ability during the tumor progression. It should therefore be regarded as a tumor suppressor and a favorable prognostic marker of patients (11) (32-34). In a previously OS study, Zhang et al. showed that ANGPTL4 promoted tumor progression, which is inconsistent with our findings (13). However, there was no more detailed mechanism research that can be referred to in Zhang's study, which remains no definite explanation for these contradictory phenomena to date. Mechanically, ANGPTL4 could be processed and cleaved into two main functional domains, the $N$-terminal fragment (nANGPTL4) and $C$-terminal fragment (cANGPTL4) $(35,36)$. The difference in function of the full-length ANGPTL4 (flANGPTL4) and the cleaved form may contribute to the contrary experimental results. Except for being directly cleaved, as a secreted glycoprotein, the diverse post-translational modification of ANGPTL4 was also a significant factor that affects the functions of ANGPTL4. For example, an abnormal sialylation of ANGPTL4 was observed in nephrotic syndrome (37). Furthermore, available data suggest that the different experimental models, 
different microenvironments, and even different cell lines could be the reasons why confounded results were observed in different studies.

Even if more research is needed if we want to understand the overall mechanism by which ANGPTL4 affects tumors, the present results revealed significant components of the whole mechanism. Recent research has shed new light on lipid metabolism and progression in tumors. Pascual et al. pointed out that a high-fat diet promotes the metastasis of oral squamous cell carcinoma and melanoma in an animal model through epigenetic regulation (38). In addition, the immune microenvironment of the intestinal tract was validated and could be regulated by a high-fat diet which enhanced intestinal tumorigenesis (39). Interestingly, previous studies had found a mutual effect between BCAAs and lipid metabolism $(40,41)$. Supplementation with BCAAs in a diet-induced obese mice model results in significant hepatic metabolic disorder that promoted gluconeogenesis and inhibited lipogenesis (24). In contrast, the suppression of the BCAA catabolism was also observed under a high-fat circumstance, and the expression of BCAA catabolism enzymes was reduced in the mice fed a high-fat diet (42). Conceivably, although the underlying mechanism is unknown to date, the interaction between BCAA metabolism and lipid metabolism will definitely affect tumor progression. According to our results, ANGPTL4 serves as not only a lipid regulator, but a bridge between BCAA metabolism and lipid metabolism as well.

Although our current results have partly clarified the organization and relationship of ANGPTL4, BCAA metabolism, and mTOR signaling pathways in the progression of OS, the precise mechanisms involved in this loop remain unclear and need to be further studied. In particular, we better understand how ANGPTL4 regulates metabolism of BCAAs, thereby regulating tumor progression of OS through the activation of mTOR signaling pathway, but we do not know exactly how various receptors are involved. The precise mechanisms involved in this loop especially the cascade between the ANGPTL4 and the BCATs were unclear and need to be further studied. Furthermore, the present results demonstrate the significant role of ANGPTL4 in the progression of OS. However, no more details were obtained, and it is still unclear whether it is the fIANGPTL4, the truncated ANGPTL4 (nANGPTL4, cANGPTL4) or the coaction of all three that plays a role in tumor progression. Thus, additional studies are needed to further clarify the exact mechanism underlying the BCAA metabolism alteration after ANGPTL4 regulation during OS progression.

In summary, we discovered that low levels of expression of ANGPTL4 regulated the metabolism of BCAAs to activate the mTOR signaling pathway, leading to accelerated proliferation in OS, as presented in Fig. 7. ANGPTL4 may be a reasonable and not previously reported bridge that links BCAA metabolism and lipid metabolism to promote OS progression. Given that overexpression of ANGPTL4 reduced the progression of OS, increasing the expression of ANGPTL4 in tumor cells of patients with OS may be a promising therapeutic strategy for OS in the future.

\section{Declarations}

Ethics approval and consent to participate

Page $11 / 23$ 
This study was approved by the Ethics Committee of Shanghai Sixth People's Hospital

\section{Consent for publication}

All authors involved in the authorship are consent for publication in the current form.

\section{Availability of data and materials}

The datasets supporting the conclusions of this article are included within the article (and its additional files).

\section{Competing interests}

The authors declare that the research was conducted in the absence of any commercial or financial relationships that could be construed as a potential conflict of interest.

\section{Funding}

This work was supported by the National Natural Science Foundation of China (NNSFC) (grant no. 81572239).

\section{Authors' contributions}

LSY carried out the experiment and wrote the manuscript; MY and ZX carried out the experiment; DY and YQC prepared the clinical samples; YQJ quantified and analyzed the data; XJ, ZSM and YT designed the research, reviewed and edited the manuscript. All authors contributed to manuscript revision, read, and approved the submitted version.

\section{Acknowledgements}

The authors would like to thank the Institute of Microsurgery on Extremities, Shanghai Jiao Tong University Affiliated Sixth People's Hospital, and the Laboratory Animal Research Center of Shanghai Sixth People's Hospital for providing experimental platform.

\section{References}

1. Gianferante DM, Mirabello L, Savage SA. Germline and somatic genetics of osteosarcoma connecting aetiology, biology and therapy. Nature reviews Endocrinology. 2017;13(8):480-91. 
2. Ritter J, Bielack SS. Osteosarcoma. Annals of oncology : official journal of the European Society for Medical Oncology. 2010;21 Suppl 7:vii320-5.

3. Kansara M, Teng MW, Smyth MJ, Thomas DM. Translational biology of osteosarcoma. Nature reviews Cancer. 2014;14(11):722-35.

4. Song BS, Seo J, Kim DH, Lim JS, Yoo JY, Lee JA. Gemcitabine and docetaxel for the treatment of children and adolescents with recurrent or refractory osteosarcoma: Korea Cancer Center Hospital experience. Pediatric blood \& cancer. 2014;61(8):1376-81.

5. Duffaud F, Egerer G, Ferrari S, Rassam H, Boecker U, Bui-Nguyen B. A phase II trial of second-line pemetrexed in adults with advanced/metastatic osteosarcoma. European journal of cancer (Oxford, England : 1990). 2012;48(4):564-70.

6. Santulli G. Angiopoietin-like proteins: a comprehensive look. Front Endocrinol (Lausanne). 2014;5:4.

7. Aryal B, Price NL, Suarez Y, Fernández-Hernando C. ANGPTL4 in Metabolic and Cardiovascular Disease. Trends in molecular medicine. 2019;25(8):723-34.

8. La Paglia L, Listì A, Caruso S, Amodeo V, Passiglia F, Bazan V, et al. Potential Role of ANGPTL4 in the Cross Talk between Metabolism and Cancer through PPAR Signaling Pathway. PPAR research. 2017;2017:8187235.

9. Fernández-Hernando C, Suárez Y. ANGPTL4: a multifunctional protein involved in metabolism and vascular homeostasis. Current opinion in hematology. 2020;27(3):206-13.

10. Chen JW, Luo YJ, Yang ZF, Wen LQ, Huang L. Knockdown of angiopoietin-like 4 inhibits the development of human gastric cancer. Oncol Rep. 2018;39(4):1739-46.

11. Cai YC, Yang H, Wang KF, Chen TH, Jiang WQ, Shi YX. ANGPTL4 overexpression inhibits tumor cell adhesion and migration and predicts favorable prognosis of triple-negative breast cancer. BMC Cancer. 2020;20(1):878.

12. Tsai YT, Wu AC, Yang WB, Kao TJ, Chuang JY, Chang WC, et al. ANGPTL4 Induces TMZ Resistance of Glioblastoma by Promoting Cancer Stemness Enrichment via the EGFR/AKT/4E-BP1 Cascade. Int J Mol Sci. 2019;20(22).

13. Zhang T, Kastrenopoulou A, Larrouture Q, Athanasou NA, Knowles HJ. Angiopoietin-like 4 promotes osteosarcoma cell proliferation and migration and stimulates osteoclastogenesis. BMC Cancer. 2018;18(1):536.

14. Zhang S, Zeng X, Ren M, Mao X, Qiao S. Novel metabolic and physiological functions of branched chain amino acids: a review. J Anim Sci Biotechnol. 2017;8:10.

15. Peng $\mathrm{H}$, Wang $\mathrm{Y}$, Luo W. Multifaceted role of branched-chain amino acid metabolism in cancer. Oncogene. 2020;39(44):6747-56.

16. Lebourgeois S, Fraisse A, Hennechart-Collette C, Guillier L, Perelle S, Martin-Latil S. Development of a Real-Time Cell Analysis (RTCA) Method as a Fast and Accurate Method for Detecting Infectious Particles of the Adapted Strain of Hepatitis A Virus. Front Cell Infect Microbiol. 2018;8:335. 
17. Shimosato Y, Kameya T, Nagai K, Hirohashi S, Koide T, Hayashi H, et al. Transplantation of human tumors in nude mice. Journal of the National Cancer Institute. 1976;56(6):1251-60.

18. Wolfson RL, Chantranupong L, Saxton RA, Shen K, Scaria SM, Cantor JR, et al. Sestrin2 is a leucine sensor for the mTORC1 pathway. Science. 2016;351(6268):43-8.

19. Hu LY, Boxer PA, Kesten SR, Lei HJ, Wustrow DJ, Moreland DW, et al. The design and synthesis of human branched-chain amino acid aminotransferase inhibitors for treatment of neurodegenerative diseases. Bioorg Med Chem Lett. 2006;16(9):2337-40.

20. Ananieva EA, Wilkinson AC. Branched-chain amino acid metabolism in cancer. Current opinion in clinical nutrition and metabolic care. 2018;21(1):64-70.

21. Mayers JR, Wu C, Clish CB, Kraft P, Torrence ME, Fiske BP, et al. Elevation of circulating branchedchain amino acids is an early event in human pancreatic adenocarcinoma development. Nature medicine. 2014;20(10):1193-8.

22. Hattori A, Tsunoda M, Konuma T, Kobayashi M, Nagy T, Glushka J, et al. Cancer progression by reprogrammed BCAA metabolism in myeloid leukaemia. Nature. 2017;545(7655):500-4.

23. Mayers JR, Torrence ME, Danai LV, Papagiannakopoulos T, Davidson SM, Bauer MR, et al. Tissue of origin dictates branched-chain amino acid metabolism in mutant Kras-driven cancers. Science. 2016;353(6304):1161-5.

24. Zhao H, Zhang F, Sun D, Wang X, Zhang X, Zhang J, et al. Branched-Chain Amino Acids Exacerbate Obesity-Related Hepatic Glucose and Lipid Metabolic Disorders via Attenuating Akt2 Signaling. Diabetes. 2020;69(6):1164-77.

25. Zhang S, Lin X, Hou Q, Hu Z, Wang Y, Wang Z. Regulation of mTORC1 by amino acids in mammalian cells: A general picture of recent advances. Anim Nutr. 2021;7(4):1009-23.

26. Shao D, Villet O, Zhang Z, Choi SW, Yan J, Ritterhoff J, et al. Glucose promotes cell growth by suppressing branched-chain amino acid degradation. Nat Commun. 2018;9(1):2935.

27. Ericksen RE, Lim SL, McDonnell E, Shuen WH, Vadiveloo M, White PJ, et al. Loss of BCAA Catabolism during Carcinogenesis Enhances mTORC1 Activity and Promotes Tumor Development and Progression. Cell metabolism. 2019;29(5):1151-65.e6.

28. Gu Z, Liu Y, Cai F, Patrick M, Zmajkovic J, Cao H, et al. Loss of EZH2 Reprograms BCAA Metabolism to Drive Leukemic Transformation. Cancer Discov. 2019;9(9):1228-47.

29. Lei MZ, Li XX, Zhang Y, Li JT, Zhang F, Wang YP, et al. Acetylation promotes BCAT2 degradation to suppress BCAA catabolism and pancreatic cancer growth. Signal transduction and targeted therapy. 2020;5(1):70.

30. Shen CJ, Chang KY, Lin BW, Lin WT, Su CM, Tsai JP, et al. Oleic acid-induced NOX4 is dependent on ANGPTL4 expression to promote human colorectal cancer metastasis. Theranostics. 2020;10(16):7083-99.

31. Nie D, Zheng Q, Liu L, Mao X, Li Z. Up-regulated of Angiopoietin-Like Protein 4 Predicts Poor Prognosis in Cervical Cancer. Journal of Cancer. 2019;10(8):1896-901. 
32. Galaup A, Cazes A, Le Jan S, Philippe J, Connault E, Le Coz E, et al. Angiopoietin-like 4 prevents metastasis through inhibition of vascular permeability and tumor cell motility and invasiveness. Proceedings of the National Academy of Sciences of the United States of America. 2006;103(49):18721-6.

33. Ito Y, Oike Y, Yasunaga K, Hamada K, Miyata K, Matsumoto S, et al. Inhibition of angiogenesis and vascular leakiness by angiopoietin-related protein 4. Cancer research. 2003;63(20):6651-7.

34. Okochi-Takada E, Hattori N, Tsukamoto T, Miyamoto $\mathrm{K}$, Ando $\mathrm{T}$, Ito $\mathrm{S}$, et al. ANGPTL4 is a secreted tumor suppressor that inhibits angiogenesis. Oncogene. 2014;33(17):2273-8.

35. Ge H, Yang G, Huang L, Motola DL, Pourbahrami T, Li C. Oligomerization and regulated proteolytic processing of angiopoietin-like protein 4. J Biol Chem. 2004;279(3):2038-45.

36. Lei X, Shi F, Basu D, Huq A, Routhier S, Day R, et al. Proteolytic processing of angiopoietin-like protein 4 by proprotein convertases modulates its inhibitory effects on lipoprotein lipase activity. $J$ Biol Chem. 2011;286(18):15747-56.

37. Clement LC, Avila-Casado C, Macé C, Soria E, Bakker WW, Kersten S, et al. Podocyte-secreted angiopoietin-like-4 mediates proteinuria in glucocorticoid-sensitive nephrotic syndrome. Nature medicine. 2011;17(1):117-22.

38. Pascual G, Domínguez D, Elosúa-Bayes M, Beckedorff F, Laudanna C, Bigas C, et al. Dietary palmitic acid promotes a prometastatic memory via Schwann cells. Nature. 2021;599(7885):485-90.

39. Beyaz S, Chung C, Mou H, Bauer-Rowe KE, Xifaras ME, Ergin I, et al. Dietary suppression of MHC class II expression in intestinal epithelial cells enhances intestinal tumorigenesis. Cell Stem Cell. 2021;28(11):1922-35.e5.

40. Gannon NP, Schnuck JK, Vaughan RA. BCAA Metabolism and Insulin Sensitivity - Dysregulated by Metabolic Status? Mol Nutr Food Res. 2018;62(6):e1700756.

41. Newgard CB. Interplay between lipids and branched-chain amino acids in development of insulin resistance. Cell metabolism. 2012;15(5):606-14.

42. Estrada-Alcalde I, Tenorio-Guzman MR, Tovar AR, Salinas-Rubio D, Torre-Villalvazo I, Torres N, et al. Metabolic Fate of Branched-Chain Amino Acids During Adipogenesis, in Adipocytes From Obese Mice and C2C12 Myotubes. J Cell Biochem. 2017;118(4):808-18.

\section{Figures}


A
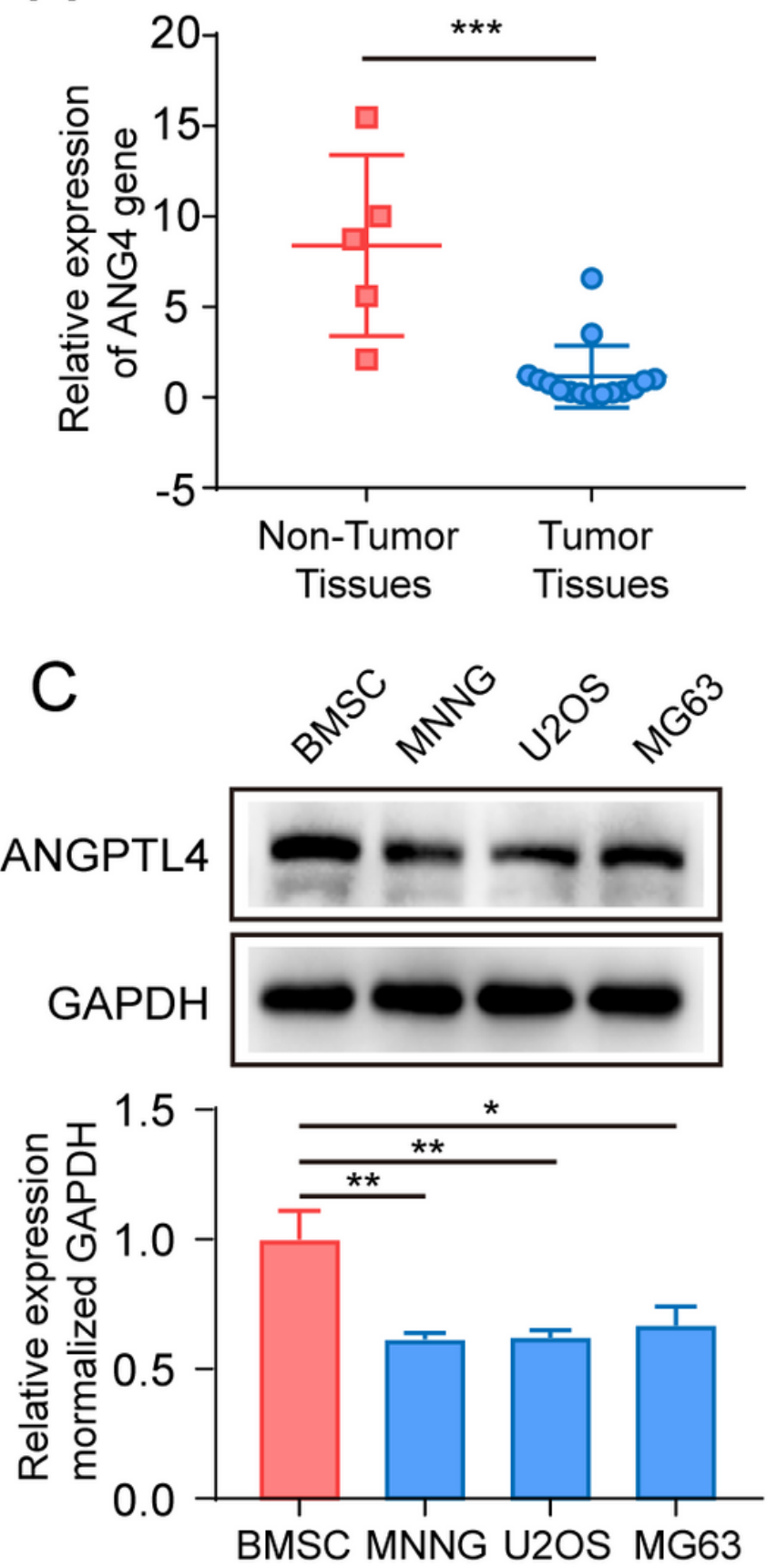

B

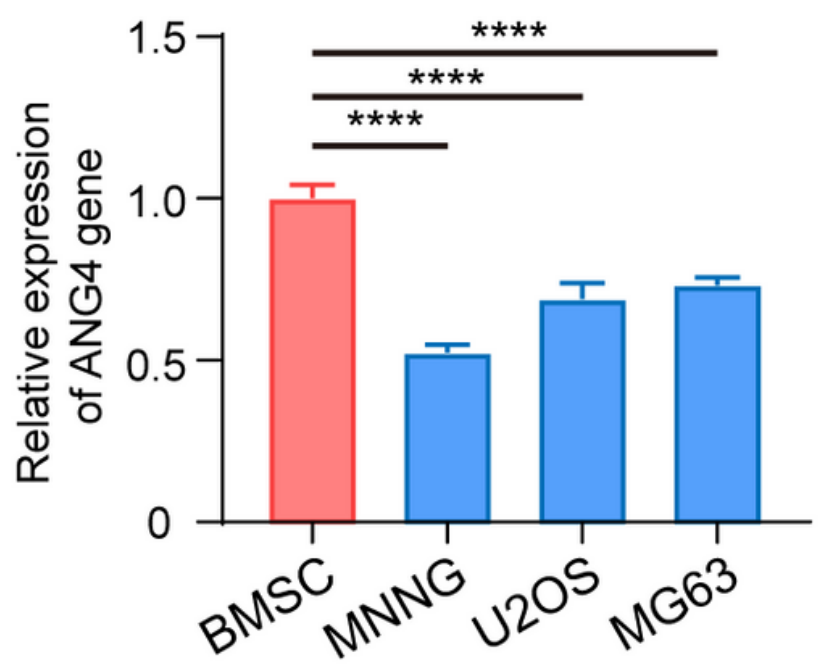
$10 \mathrm{x} \quad 40 \mathrm{X}$

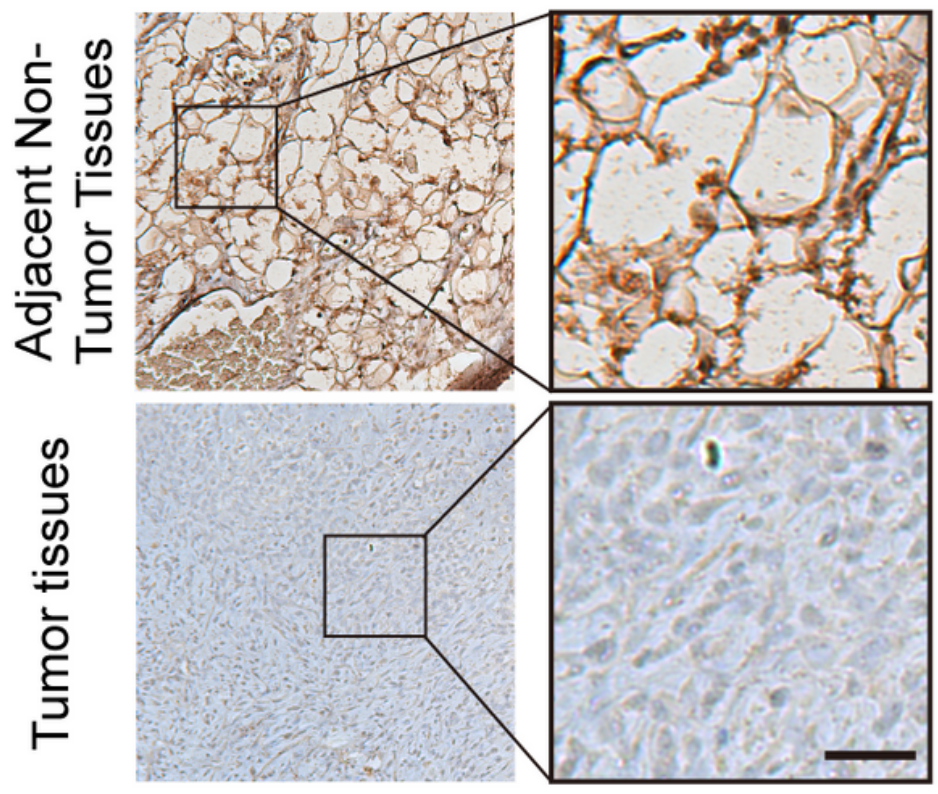

Figure 1

Expression of ANGPTL4 is lower in osteosarcoma tissue and osteosarcoma cell lines. (A) Relative expression of ANGPTL4 mRNA in clinical osteosarcoma (OS) tissue samples ( $N=15)$ and adjacent nontumor cancellous bone tissue samples $(N=5)$. (B) Relative expression of ANGPTL4 mRNA in OS cell lines (MNNG, U2OS, MG63) and the bone marrow derived stroma cell (BMSC). Mean $\pm S D(N=3)$. (C) ANGPTL4 expression (upper panels) and quantitation of protein levels (lower bar graphs) in OS cell lines and BMSC cells were detected by western blotting. Mean \pm SD $(N=3)$. (D) Representative immunohistochemical images of ANGPTL4 in clinical OS sample and adjacent nontumor bone marrow

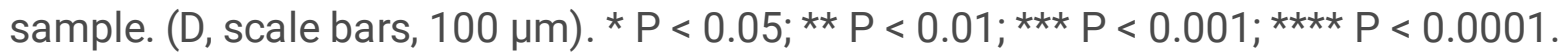




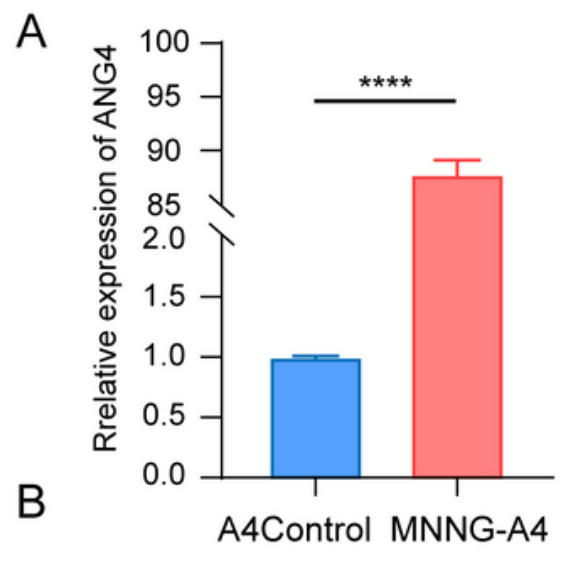

E

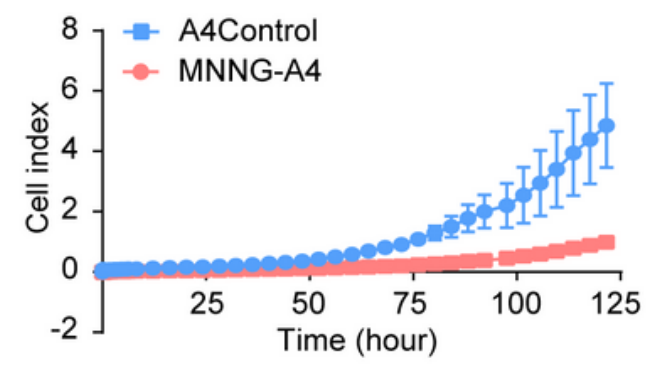

A4Control MNNG-A4

$\mathrm{F}$

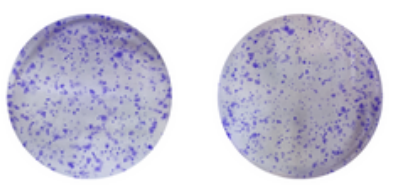

$1 \mathrm{~cm}$

J

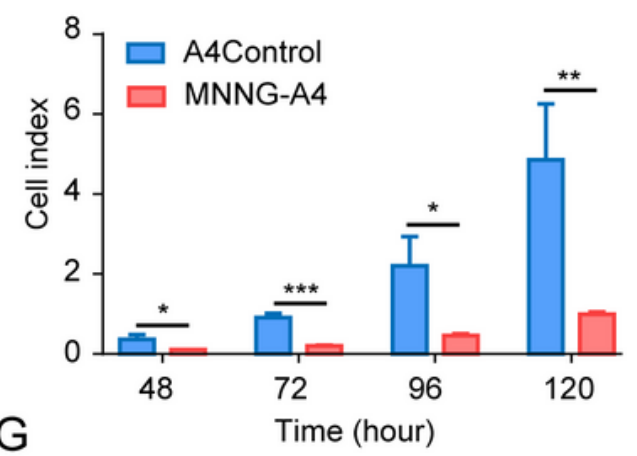

C

A4Control MNNG-A4

ANGPTL4

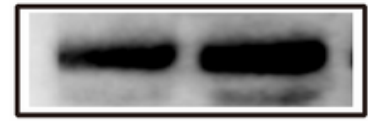

GAPDH

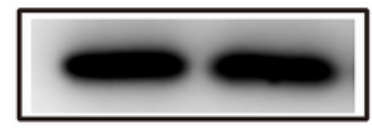

G
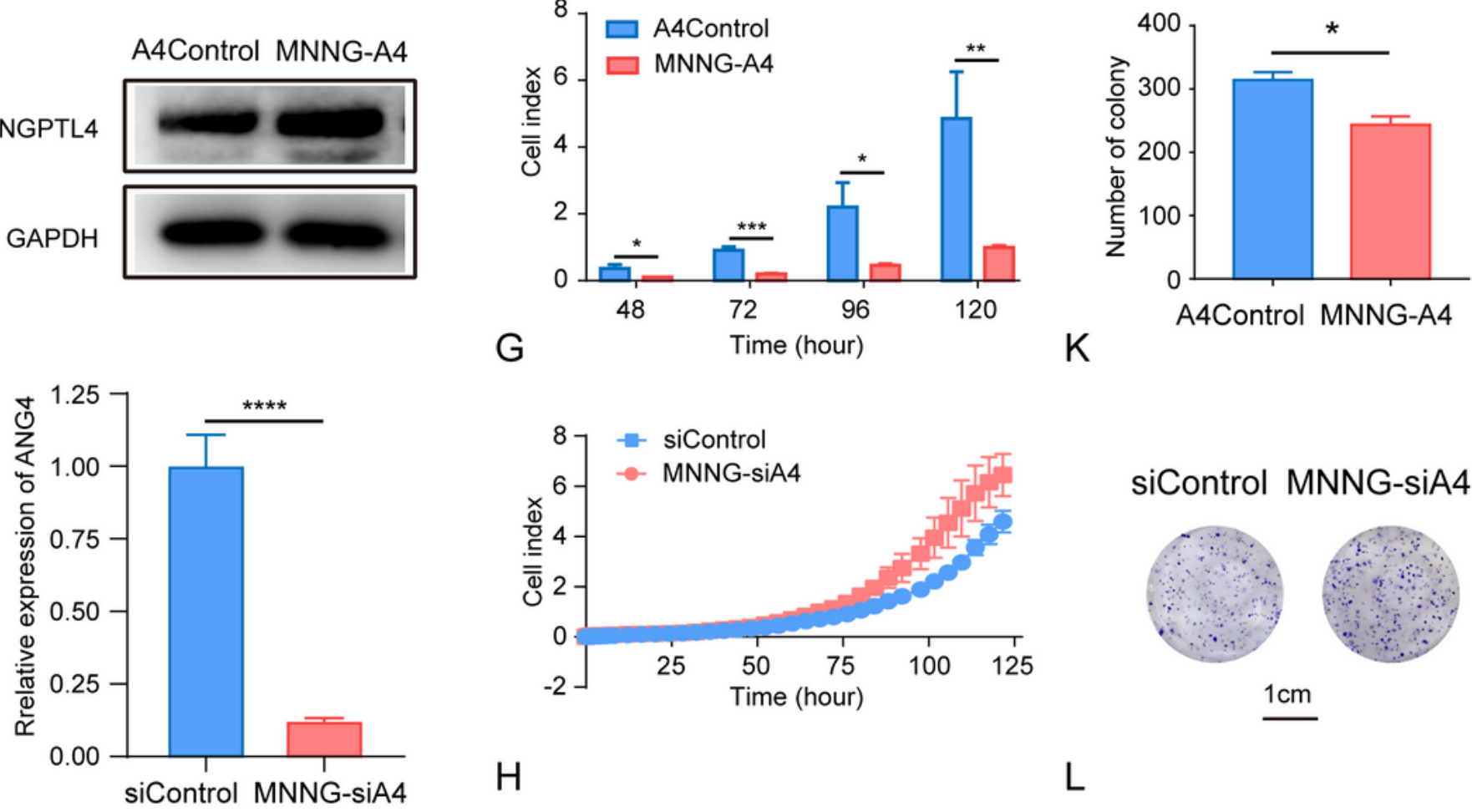

$\mathrm{K}$

siControl MNNG-siA4

$\mathrm{H}$

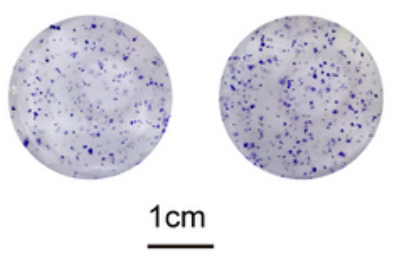

L

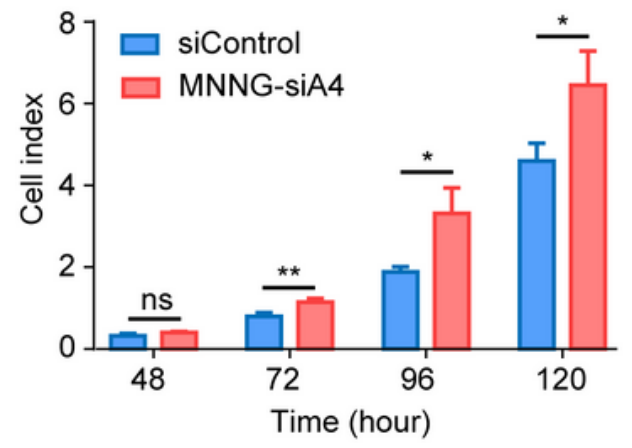

\section{Figure 2}

The Knockdown of ANGPTL4 promotes the growth of MNNG in vitro. (A-D) Quantitation of ANGPTL4 mRNA and ANGPTL4 protein in MNNG-A4, MNNG-siA4, and control cell lines by qRT-PCR and western blot, respectively. $(E, G)$ In vitro measurement of cell proliferation in two ANGPTL4-regulated MNNG cell lines (MNNG-A4, MNNG-siA4) and according control cell lines. Cell index results reflects that cell proliferation is enhanced in MNNG-siA4 cells, while reduced in MNNG-A4 cells compared to control 
groups. Mean \pm SD $(N=4)$. $(F, H)$ Cell index at 48 h, $72 h, 96 h, 120$ h. Mean $\pm S D(N=4)$. $(I, K)$

Representative images of colony formation assay in MNNG-A4, MNNG-siA4, and control cells. $(\mathrm{J}, \mathrm{L})$

Quantitation of colony counts after 2 weeks of in vitro maintenance. Mean $\pm S D(N=3)$. Mean $\pm S D(N=5)$.

Mean \pm SD. ns $P>0.05 ; * P<0.05 ; * \star P<0.01 ; * \star \star P<0.001$.

A

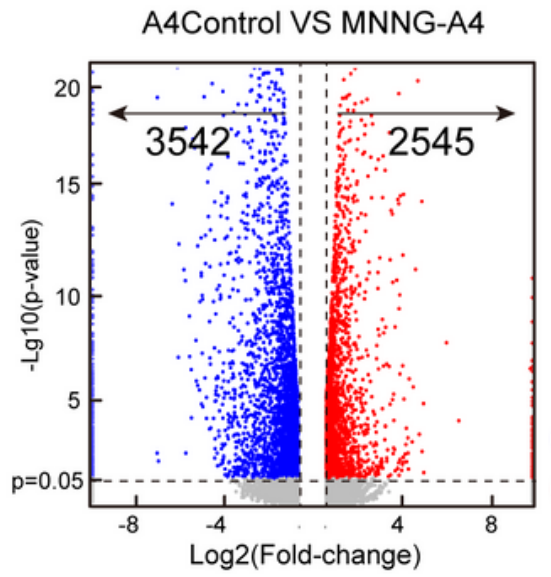

B

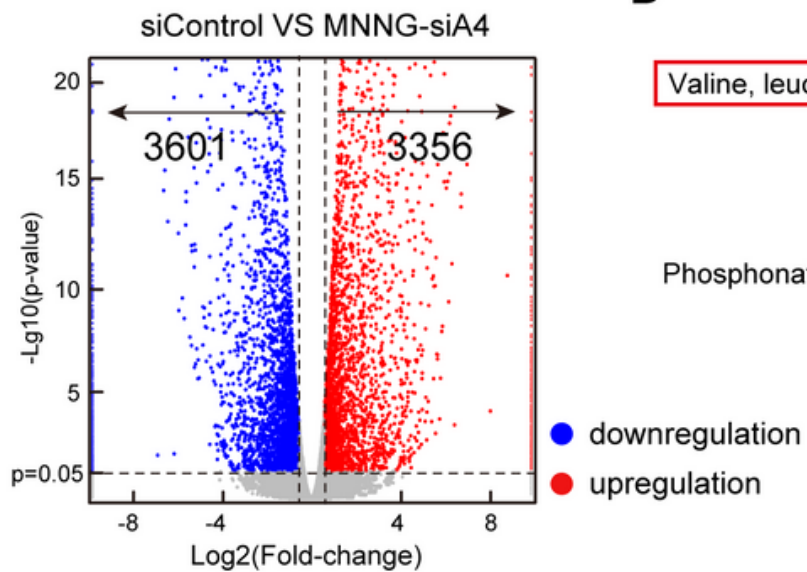

E

$\begin{array}{cccccccccc} & \bigcirc & \odot & \odot & 0 & 0 & \odot & \bigcirc & \bigcirc & \bigcirc \\ -1.50 & -1.20 & -0.90 & -0.60 & -0.30 & 0.30 & 0.60 & 0.90 & 1.20 & 1.50\end{array}$

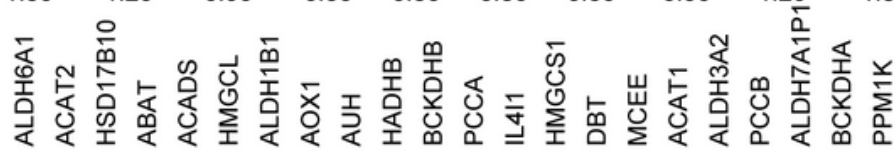
온

$\sum_{\sum}^{+}$

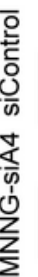

upregulation
C

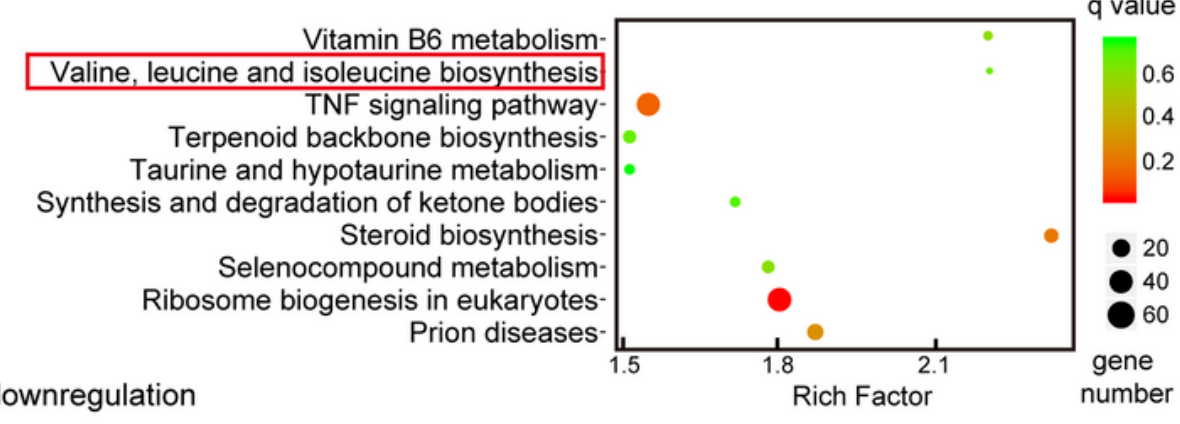

A4Control VS MNNG-A4 
The signaling discovery of ANGPTL4 functioning on OS growth by RNA-seq. (A, B) Volcano plots of the differentially expressed genes (DEGs) in MNNG-A4 cells, MNNG-siA4 cells, and their control cells. Foldchange values on the $\mathrm{X}$-axis were log2-transformed while $P$ values on the $\mathrm{Y}$-axis were-log10 transformed. The screening criteria for EDGs are positioned as $P$ value $<0.05$, and the expression level is higher than 1.5 folds or lower than 0.67 folds. (C, D) KEGG pathway enrichment analysis for DEGs in MNNG-A4 cells, MNNG-siA4 cells, and their control cells. (E) Heatmap of the BCAAs associated DEGs between ANGPTL4regulated cells and control cells. $(F)$ Protein-protein interaction network provided interactive information among the BCAAs associated DEGs. Mean $\pm \mathrm{SD}(N=3) \mathrm{ns} \mathrm{P}>0.05 ;{ }^{*} \mathrm{P}<0.05 ; * \star \mathrm{P}<0.01 ; * \star \star \mathrm{P}<0.001$.
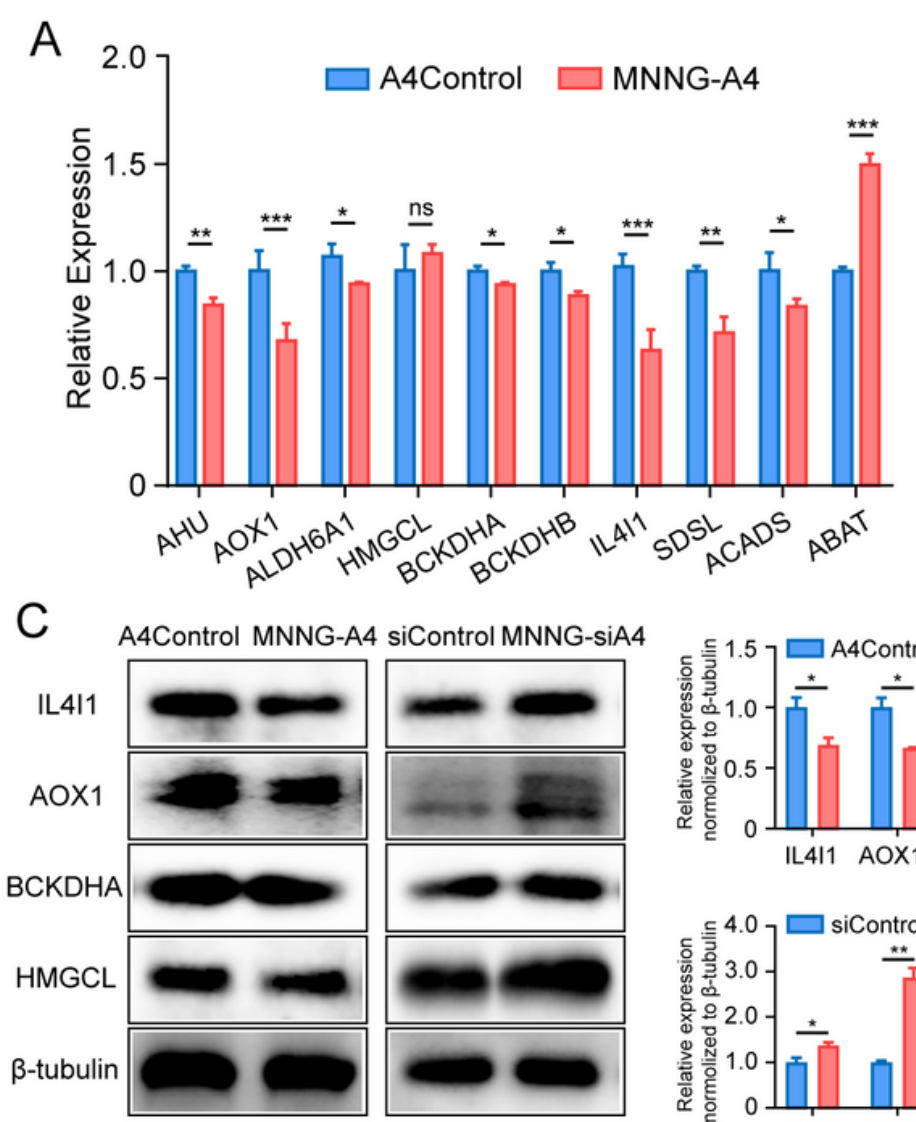

D

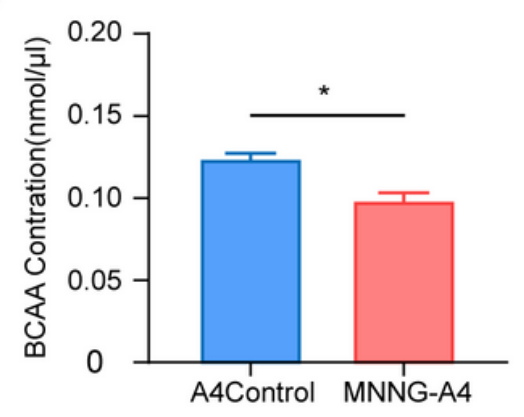

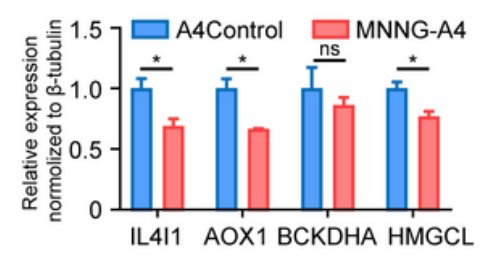
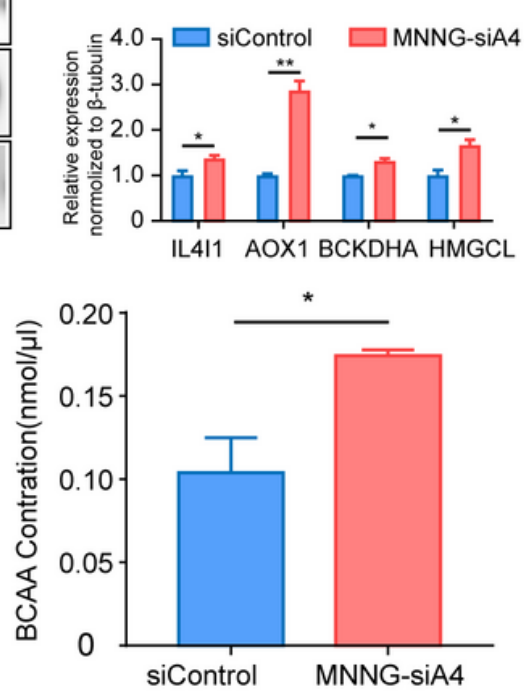

B

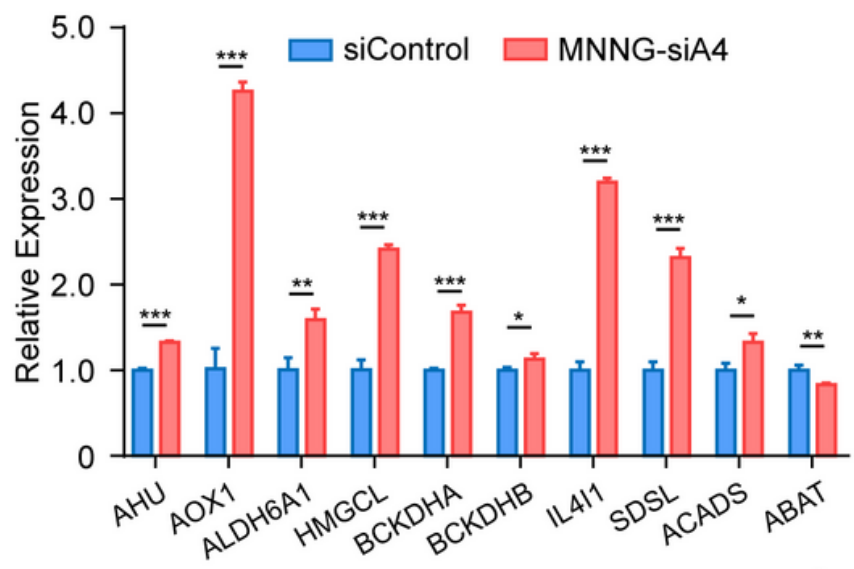

$\mathrm{E}$
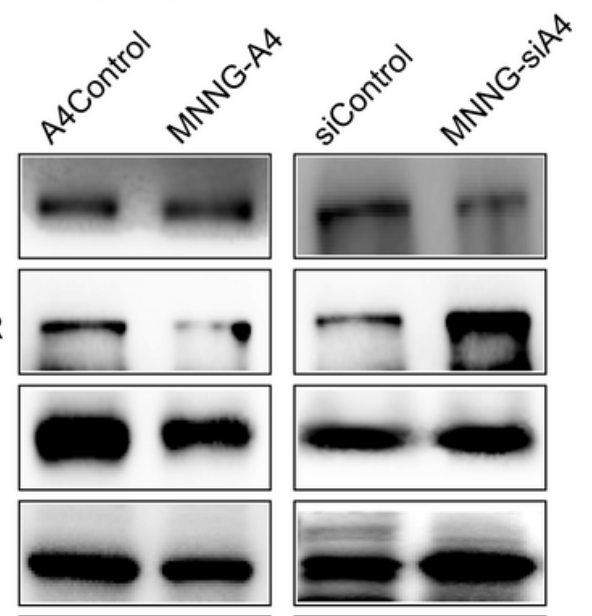

$\mathrm{m}-\mathrm{TOR}$

p-m-TOR

S6K

$\mathrm{p}-\mathrm{S} 6 \mathrm{~K}$

S6
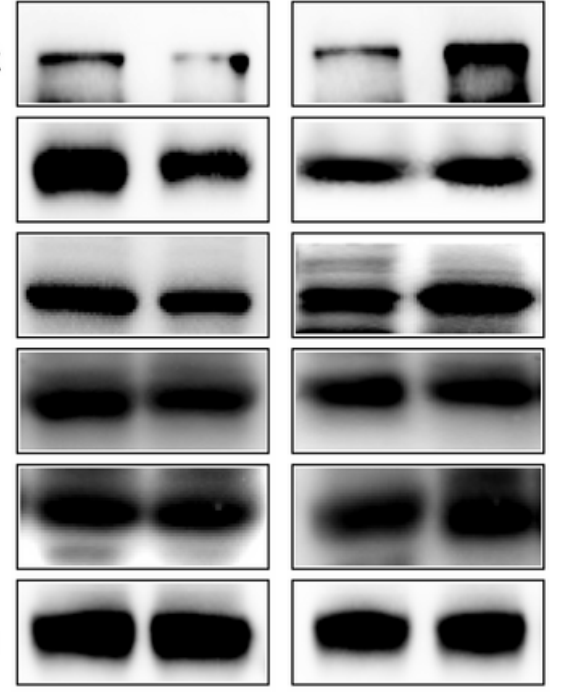

\section{Figure 4}

The low expression of ANGPTL4 promoted the accumulation of branched chain amino acid (BCAA) and activate mTOR signaling. (A, B) Among the DEGs, genes that are highly related to BCAA metabolism were verified by RT-qPCR in the ANGPTL4-regulated cells and their controls. (C) Proteins that are highly related 
to the BCAA metabolism were verified by western blotting in the ANGPTL4-regulated cells and their controls. Quantitation of relative expression. Mean $\pm S D(N=3)$. (D) The relative levels of BCAAs in MNNGA4 cells, MNNG-siA4 cells, and their control cells. (E) western blot detected proteins in the mTOR signaling pathway show that this pathway was activated in MNNG-siA4 cells but inhibited in MNNG-A4 cells. Mean $\pm \mathrm{SD}(N=3)$. ns $\mathrm{P}>0.05 ; * \mathrm{P}<0.05 ; * \star \mathrm{P}<0.01 ; * \star * \mathrm{P}<0.001$.

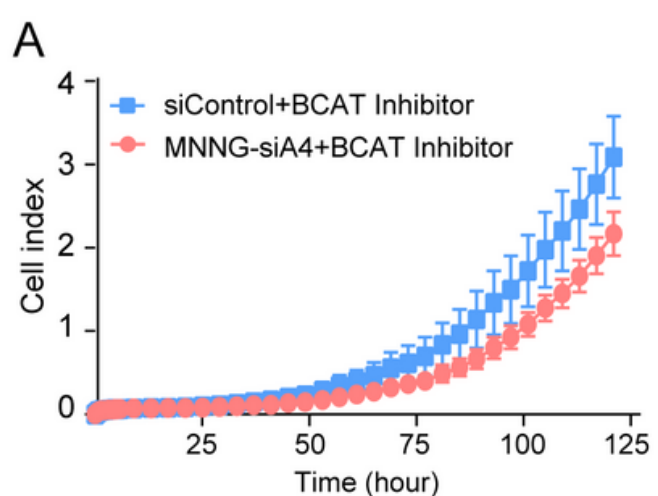

$E$

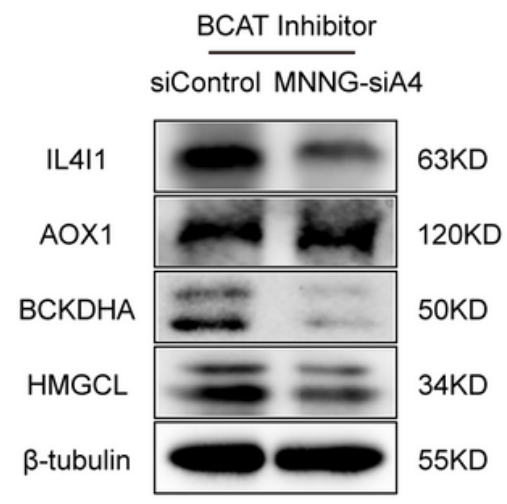

B
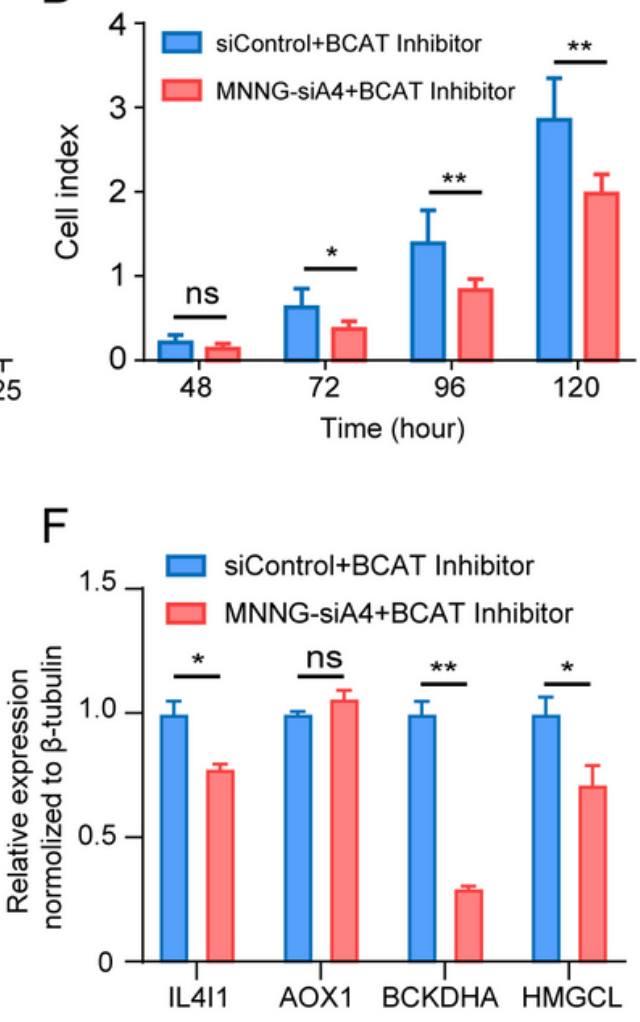

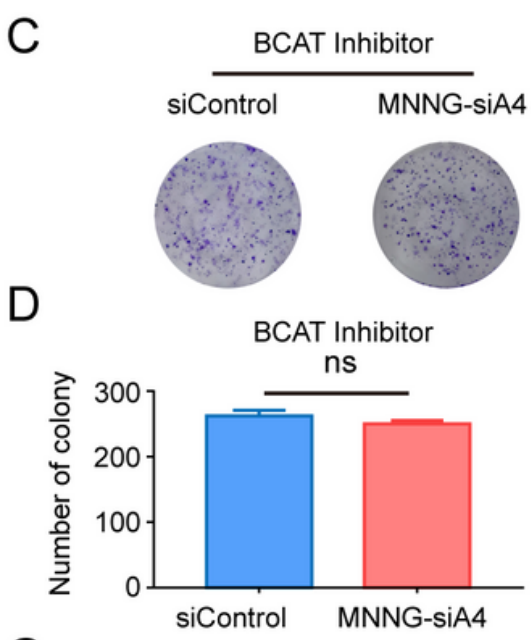

G

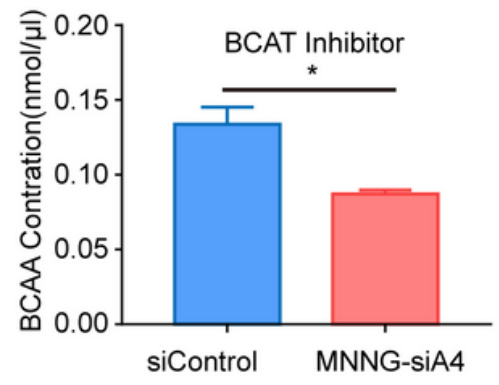

\section{Figure 5}

BCATs inhibitor attenuates the elevated growth of MNNG-siA4 cells. (A) In vitro measurement of cell attachment and cell proliferation in MNNG-siA4 and control cell lines with $5 \mu \mathrm{M}$ BCATs inhibitor treatment. (B) Cell index at $48 \mathrm{~h}, 72 \mathrm{~h}, 96 \mathrm{~h}, 120 \mathrm{~h}$ with $5 \mu \mathrm{M}$ BCATs inhibitor treatment. Mean $\pm \mathrm{SD}(N=4)$. (C) Representative images of colony formation assay in MNNG-siA4 and control cells after $5 \mu \mathrm{M}$ BCATs inhibitor treatment. (D) Quantitation of colony counts after 2 weeks of in vitro maintenance with $5 \mu \mathrm{M}$ BCATs inhibitor treatment. Mean $\pm S D(N=3)$. $(E, F)$ Proteins that are highly related to the BCAA metabolism were verified by western blotting in the MNNG-siA4 cells and control cells after $5 \mu \mathrm{M}$ BCATs inhibitor treatment. (G) The relative levels of BCAAs in MNNG-siA4 cells and control cells after $5 \mu \mathrm{M}$ BCATs inhibitor treatment. ns $\mathrm{P}>0.05$; $\mathrm{P}<0.05$; $* * \mathrm{P}<0.01$. 
A

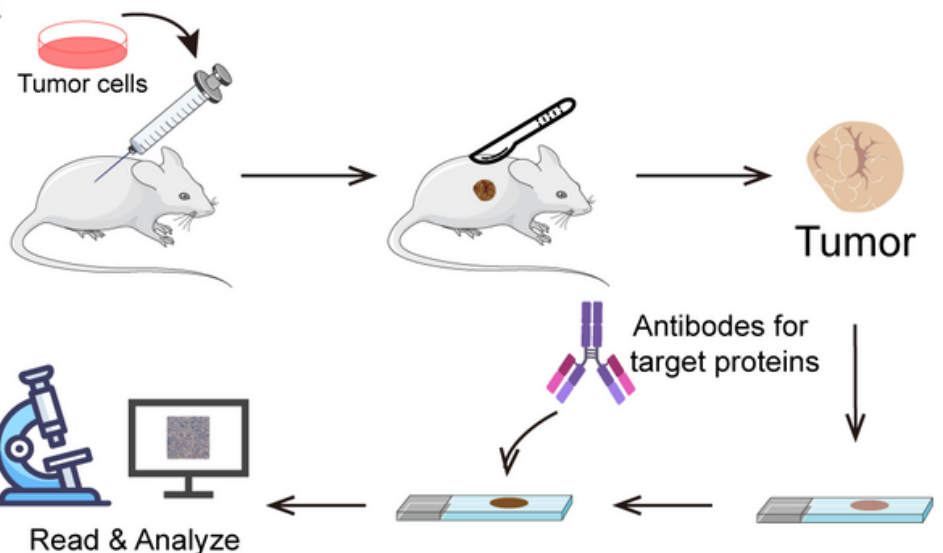

C

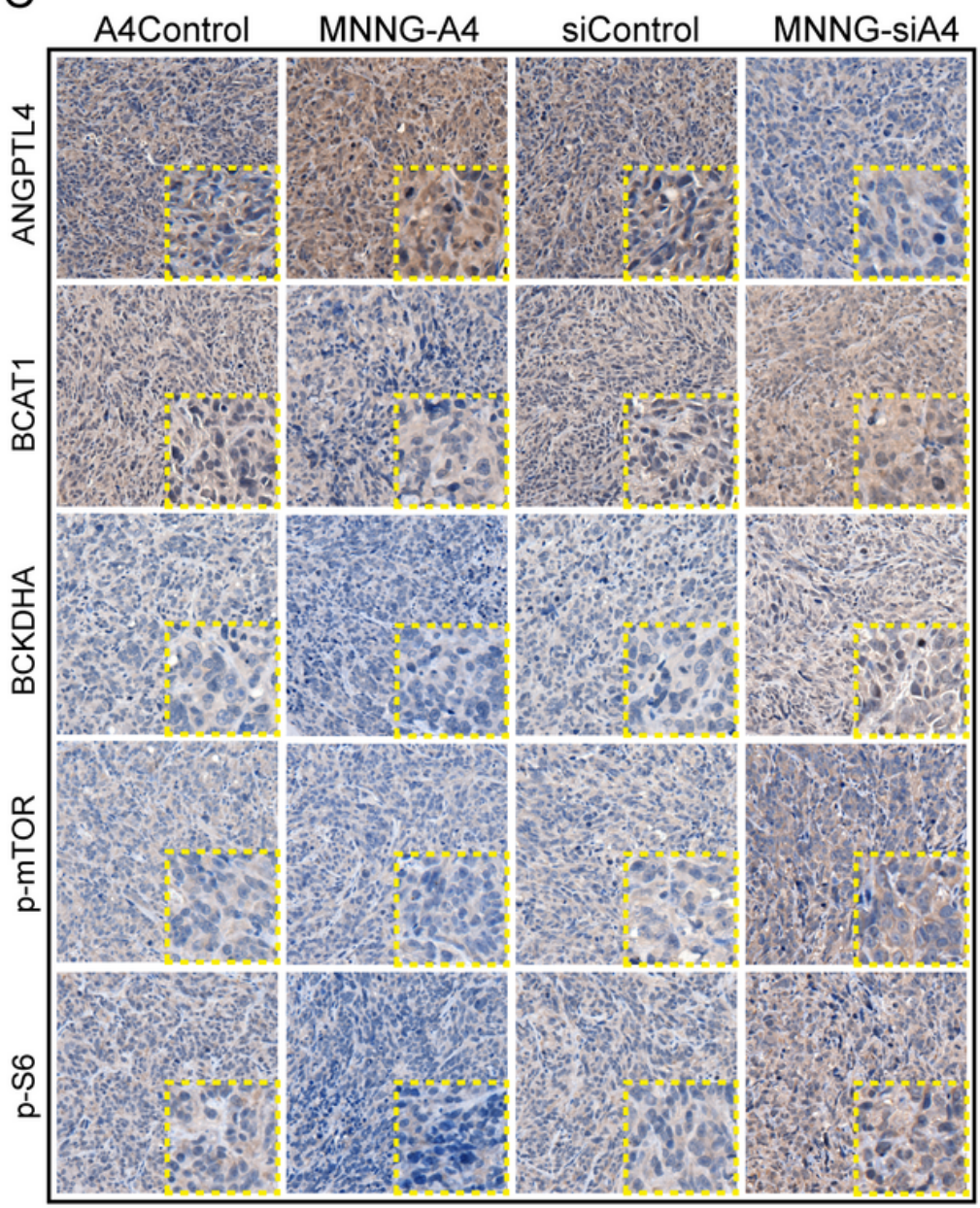

B
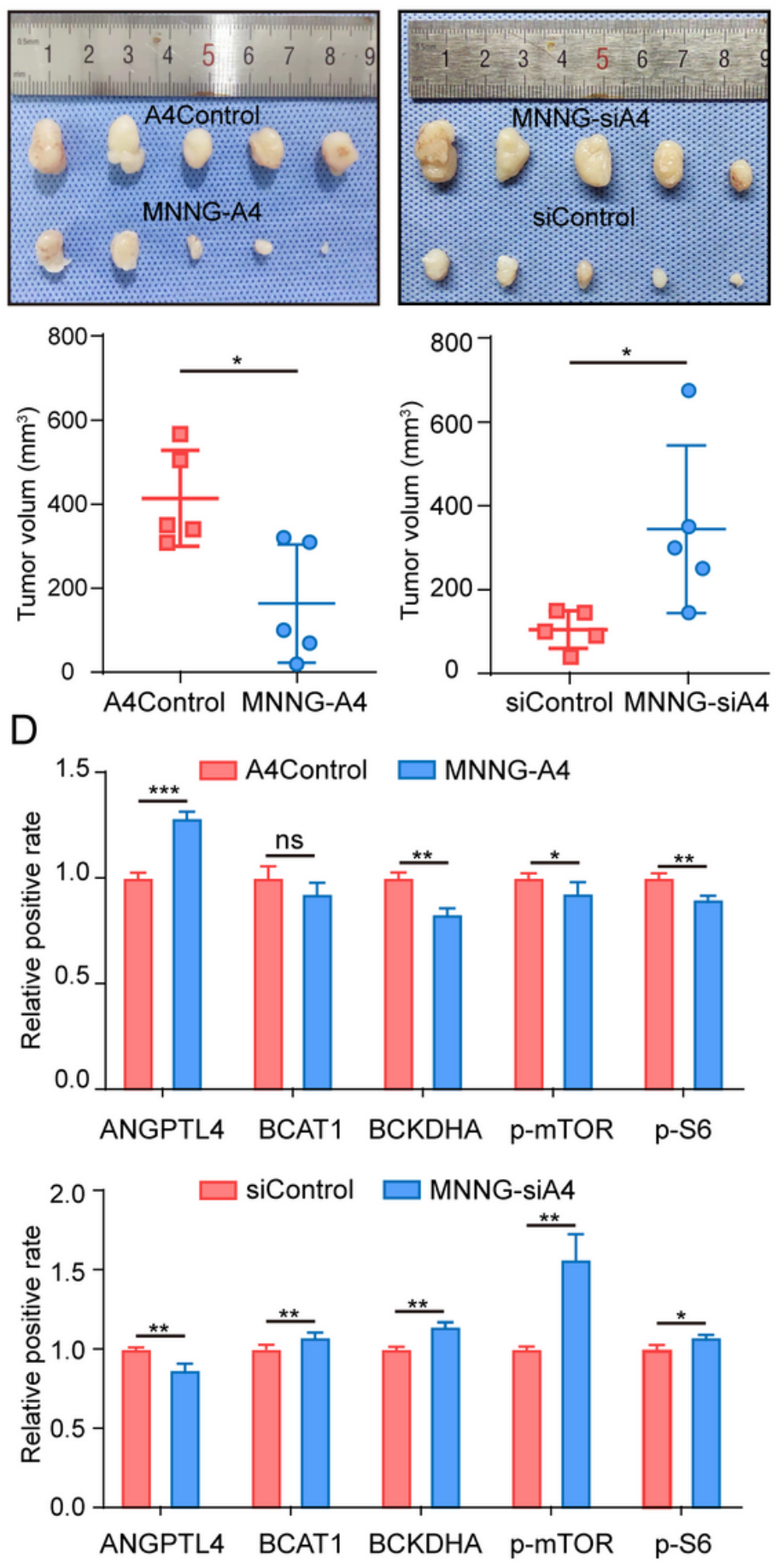

\section{Figure 6}

The expression of ANGPTL4 shows a negatively regulation with OS progress via BCAA/mTOR axis in vivo. (A) Diagram illustrating the method for constructing subcutaneous tumor model and IHC analysis. (B) Images of excised tumors from the nude mouse subcutaneous tumor model and quantitation of tumor volume. (C, D) Immunohistochemical staining of ANGPTL4, BCAT1, BCKDHA, p-mTOR, and p-S6 in xenografts of OS tissue from the subcutaneous tumor nude mouse model. 

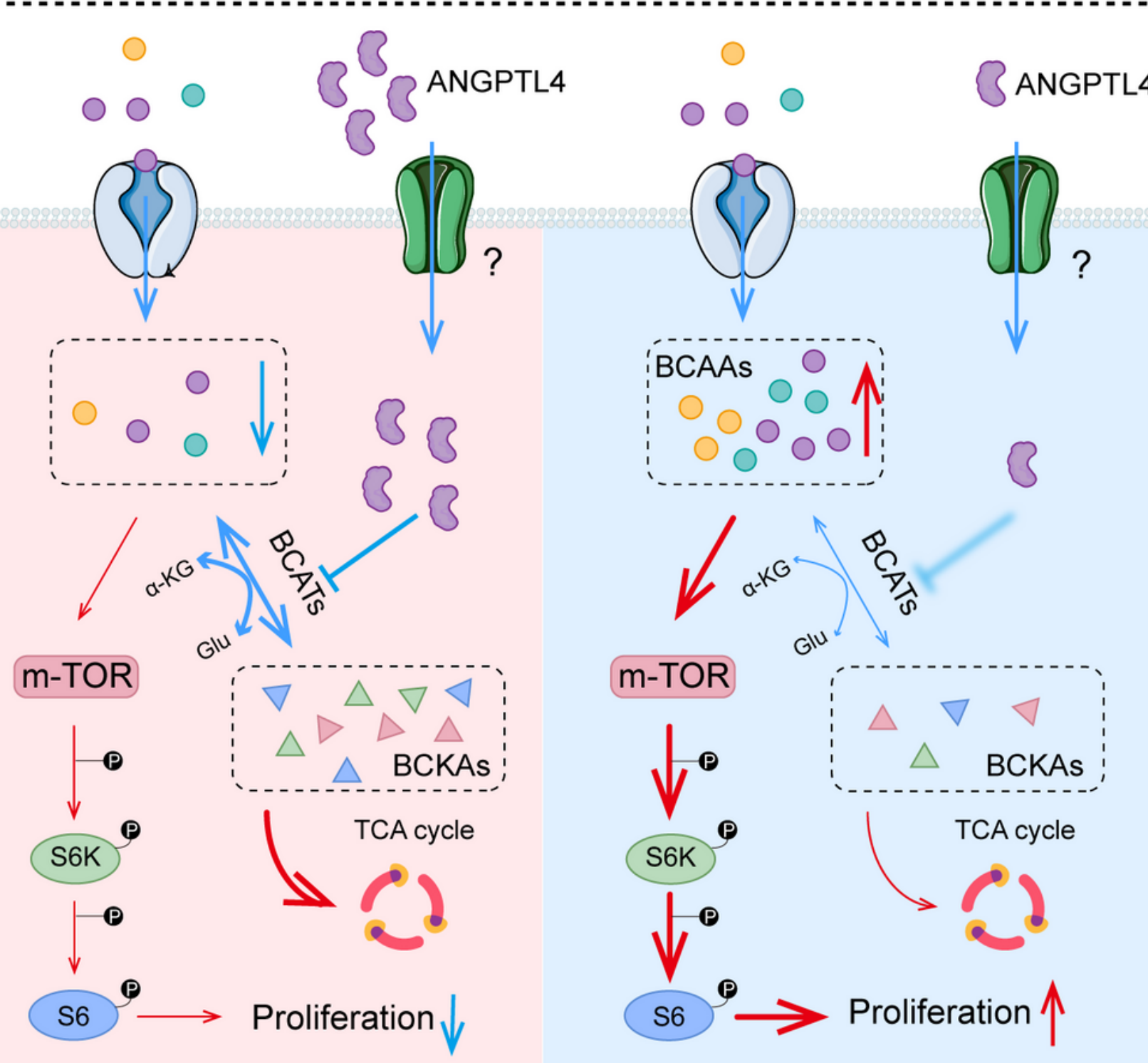

ANG4 upregulated

ANG4 downregulated

(2)

Amino acid transporters

$\sqrt{ }$ Protein transporters $\uparrow$ Promote $\rceil$ Attenuate Branched-Chain Amino Acid $\triangle$ Branched-Chain a-Keto Acids ANGPTL4

Figure 7

ANGPTL4 negatively regulates OS cell growth by remodeling BCAA metabolism. Diagram illustrating one mechanism that illustrates how ANGPTL4 influences OS cell progression through the ANGPTL4/BCAAs/mTOR axis.

\section{Supplementary Files}


This is a list of supplementary files associated with this preprint. Click to download.

- Supplementaryfiles.docx 